\title{
Efficacy and Cytokine Modulating Effects of Tacrolimus in Systemic Lupus Erythematosus: A Review
}

\author{
Kam Hon Yoon \\ El Shaddai Arthritis and Rheumatism Specialist Medical Centre, Block 102, \#01-258, Towner Road, Singapore 322102 \\ Correspondence should be addressed to Kam Hon Yoon, mdcykh@singnet.com.sg \\ Received 27 February 2010; Accepted 31 March 2010 \\ Academic Editor: Brian Poole \\ Copyright () 2010 Kam Hon Yoon. This is an open access article distributed under the Creative Commons Attribution License, \\ which permits unrestricted use, distribution, and reproduction in any medium, provided the original work is properly cited. \\ Systemic lupus erythematosus (SLE) is a complex systemic autoimmune disease with involvement of both B cells and cytotoxic \\ T lymphocytes and several cytokines aberrations. Standard therapy for SLE has its limitations. Tacrolimus, a novel calcineurin \\ inhibitor with potent immunosuppressive effects, has been shown in the recent years to be effective in SLE therapy. This paper \\ serves to collate the experimental and clinical data on the efficacy of tacrolimus in the treatment of SLE and lupus nephritis. \\ Tacrolimus as a key component of multitarget therapy in SLE is also discussed. The immunocytokine modulatory effects of \\ tacrolimus are also reviewed with reference to SLE. It can be concluded that tacrolimus has an established role in the management \\ of SLE.
}

\section{Introduction}

Systemic lupus erythematosus (SLE) is a complex systemic autoimmune disease with multiorgan involvement characterized by autoantibody formation and immune complex deposition. The pathogenesis of SLE is being unravelled and involves a complex interplay of environmental triggers, hormonal factors, susceptibility genes including genes that control apoptosis rates, antigen/immune complex clearance, lymphoid signaling, and genes that influence inflammatory responses. Examples include lpr gene (Fas mutations) in MRL mice; Clq gene polymorphism; mannose binding lectin gene polymorphism; and IL-10 gene polymorphism [1].

Both $\mathrm{B}$ cells and cytotoxic $\mathrm{T}$ lymphocytes are involved in the pathogenesis of SLE. The cytokine profile of SLE has been extensively studied. Current theory proposes an overexpression of certain $\mathrm{T}_{\mathrm{H}} 2$ cytokines that suppress the $\mathrm{T}_{\mathrm{H}} 1$ response in lupus T-cells. Examples include studies in lupusprone F1 mice showing higher IFN- $\gamma$ and IL-4 levels and lower active TGF- $\beta$ levels; and in patients with SLE, serum IL-10 levels were higher than in controls and correlated with disease activity $[2,3]$.

The standard therapy for SLE includes steroids, antimalarias, azathioprine (AZA), and cytotoxic therapy with cyclophosphamide (CYC). Mycophenolate mofetil (MMF) has been shown to be useful for lupus nephritis and also recently rituximab, anti-B cell therapy has been shown to be efficacious for refractory cases. In this paper, however, I would like to focus on tacrolimus (FK506, Prograf), a relatively new calcineurin inhibitor that has been increasingly used in transplant medicine. Tacrolimus is a macrolide compound isolated from Streptomyces tsukubaensis, a soil fungus found in Northern Japan. It was first recognized for its immunosuppressive properties and used extensively in transplantation in the recent years. The objective of this paper is to summarize the reported literature concerning the clinical efficacy of tacrolimus in the management of SLE and the cytokine modulating effects of tacrolimus.

1.1. Tacrolimus in Experimental SLE Models. There were two studies involving MRL/lpr and B/W F1 mice, where tacrolimus was administered to the mice with spontaneous lupus nephritis [4]. The drug was able to reduce proteinuria and prolong the lifespan of the lupus mice and also prevented the progression of the nephropathy. Histopathological study showed that tacrolimus significantly inhibited glomerular hypercellularity and crescent formation. The elevated serum anti-ds DNA was also suppressed by tacrolimus [5]. 
1.2. Tacrolimus Therapy for SLE without Renal Involvement. Duddridge was the first to report the efficacy of tacrolimus in the treatment of 2 SLE patients with severe cutaneous vasculitis, where cyclophosphamide and cyclosporin had failed [6]. Subsequently, there were some reports of topical tacrolimus being effective for malar rash, chronic discoid lupus, and treatment-resistant cutaneous lupus erythematosus [7-9]. A recent report from Japan also successfully treated 10 SLE patients without renal involvement with tacrolimus [10]. The mean SLE Disease Activity Index (SLEDAI) significantly decreased after 1 year with a reduction of mean dose of prednisolone. The author has also reported 2 patients with SLE with antiphospholipid syndrome who were treated with tacrolimus and had improvement in the manifestations of cutaneous vasculitis and arthritis [11].

1.3. Efficacy of Tacrolimus in SLE with Renal Involvement (Lupus Nephritis). The efficacy of tacrolimus in SLE patients with lupus nephritis was first reported in 4 pediatric patients who had persistent disease activity despite conventional immunosuppressive therapy including AZA, MMF, or CYC [12]. They had marked improvement in disease indices during treatment with FK506.

The author was first to report the therapeutic success of tacrolimus in 6 adult SLE patients with relapsed lupus nephritis [11]. All 6 patients had reduction in proteinuria, having failed conventional immunosuppressive regimes.

Subsequent to these, several reports emerged regarding the usage of tacrolimus in lupus nephritis. An open-labeled pilot study using FK506 as an induction therapy for diffuse proliferative lupus nephritis in 9 patients reported by Mok et al. from Hong Kong showed complete response in $67 \%$ and $22 \%$ had partial response [13]. This was further confirmed by 2 reports from Zhang et al. where FK506 was found to be comparable to intravenous CYC in the induction therapy of class IV lupus nephritis [14].

Effectiveness of tacrolimus for membranous class V lupus nephritis was also confirmed in case reports and other case series from Japan and Hong Kong [15-17]. As compared with conventional cytotoxic treatment, tacrolimus resulted in a faster resolution of proteinuria, and a lower risk of lupus flare within 1 year.

Recent reports from Japan also showed tacrolimus at $3 \mathrm{mg} /$ day to be effective and safe for maintenance treatment up to one-year follow-up in lupus nephritis [18]. Another double-blind, placebo-controlled study in 63 patients by Nobuyuki et al. also showed that the addition of tacrolimus at $3 \mathrm{mg} /$ day to glucocorticoid therapy resulted in significant improvement in lupus nephritis compared with placebo, up to 28 weeks [19]. This was evident by reduction in the disease activity index, reduction in urinary protein excretion, and increase in complement $\mathrm{C} 3$ level.

A novel immunotherapeutic approach has been recently proposed and studied by Bao et al. for the treatment of mixed class V + IV lupus nephritis that is resistant to conventional treatment [20]. Remission rates of this subtype of lupus nephritis are low with only $21 \%$ with CTX therapy and $20 \%$ with MMF. A six-month study using tacrolimus as induction therapy for this mixed class V + IV lupus nephritis showed a response rate of $21.1 \%$ [21]. The new approach of multitarget therapy using a combination of prednisolone, MMF, and tacrolimus showed a significantly much higher complete remission at both 6 and 9 months $(50 \%$ and $65 \%$, resp.) than with IV CYC (5\% and 15\%, resp.). In addition, $40 \%$ of patients had partial remission at 6 and 9 months follow-up. Adverse events were also less frequent in the multitarget therapy group.

1.4. Cytokine Modulating Effects of Tacrolimus in SLE. The pathogenesis of SLE is complex and involves the FCreceptors system, complements, autoreactive $\mathrm{T}$ cells and increased B cell activation, signal pathway alterations, with expansion, hyperreactivity, and production of autoantibodies. A consequence of ongoing T cell stimulation is release of cytokines, and elevation of certain cytokines in SLE has been well recognized, especially during periods of clinical activity. The "lupus storm" of hyperpyrexia and vascular collapse seen more in the presteroid era of SLE might also have had a component of a cytokine release syndrome [22].

Interferon- $\alpha$ (IFN- $\alpha$ ) was the first cytokine found to be elevated in SLE and increased levels correlated with disease activity. High levels of tumour necrosis factor alpha (TNF- $\alpha$ ) have also been identified in some patients with active SLE and positively correlate with levels of circulating autoantibodies. Serum IL-6 and IL-10 are also increased in SLE patients [23].

In SLE, it appears that both the $\mathrm{T}_{\mathrm{H}} 1$ and $\mathrm{T}_{\mathrm{H}} 2$ responses are in operation. $\mathrm{A} \mathrm{T}_{\mathrm{H}} 1$ environment favours the production of autoimmunity that is characterized by $\mathrm{T}$ cell mechanisms, while autoimmunity involving antibody formation is fostered by a $\mathrm{T}_{\mathrm{H}} 2$ environment. $\mathrm{T}_{\mathrm{H}} 1$ cells characteristically produce IL-2, IFN- $\gamma$, IL- 12 , and TNF- $\alpha$, while $\mathrm{T}_{\mathrm{H}} 2$ cells secrete IL-4, IL-5, IL-6, IL-10, and IL-13.

Tacrolimus binds to FK-binding proteins (FKBPs) in the cytoplasm, and the complex associates with the calciumdependent calcineurin/calmodulin complexes to impede calcium-dependent signal transduction in lymphocytes [24]. This causes the transcription factors that promote cytokine gene activation to be reduced.

In terms of the cytokine modulating effects of tacrolimus, it potently inhibits T cell activation-induced TNF- $\alpha$ and IL$1 \beta$ production in vitro by human peripheral blood mononuclear cells (PBMCs) [25]. Tacrolimus was also found to be more potent than dexamethasone and cyclosporin A in that regards. The cytokine suppressive effects of tacrolimus have been studied in various autoimmune conditions. Both $\mathrm{T}_{\mathrm{H}} 1$ (IL-2, IFN- $\gamma$ ) and $\mathrm{T}_{\mathrm{H}} 2$ cytokines (IL-4, IL-5) were found to be suppressed by tacrolimus in atopic dermatitis [26]. In the rat adjuvant-induced arthritis model, FK506 was found to be more effective than methotrexate in reducing elevated levels of inflammatory cytokines, TNF- $\alpha$, IL-1 $\beta$, and IL-6 [27]. The suppression of IL- 6 production and IgM production by FK506 was also confirmed in human PBMC [28]. This is important in SLE therapy as IL-6 overexpression induces B cell differentiation and increases production of autoantibodies. In the myasthenia gravis model, where PBMCs of patients were cultured for cytokine profile and studied, the culture study showed reduced IL-12, IL-17, IFN- $\gamma$, GM-CSF, TNF- $\alpha$, 
and MIP- $1 \beta$ in patients who received tacrolimus [29]. This suggests that tacrolimus inhibits $\mathrm{T}$ cells and macrophages and enhances type 1 regulatory $\mathrm{T}$ cells. A recent study also confirmed that treatment with tacrolimus inhibited the expression of $\mathrm{T}_{\mathrm{H}} 1$ cytokine mRNA in lupus-prone mice [30].

In addition, tacrolimus has been shown to inhibit IL-10 production [31]. This is important as IL-10 plays an important role in the pathogenesis of SLE [32]. The deleterious effects of IL-10 include stimulation of terminal $B$ cell differentiation, activation induced cell death, and a suppressive effect on T cell. Elevated levels of IL-10 are seen in SLE patients and correlated with SLE activity.

In terms of affecting the humoral immunity response, tacrolimus does not target B cells directly but works indirectly by interfering with $\mathrm{T}$ cell help [33]. Tacrolimus decreased the expression of the costimulating ligands (CD154 and CD278), reducing the ability of T cells to activate B cells. Tacrolimus also attenuated B cell stimulatory cytokine mRNA levels in T cells, thereby abrogating B cell signals necessary for activation and class-switching. Hence, tacrolimus is able to inhibit T-cell-dependent immunoglobulin production.

The ability of tacrolimus to affect $\mathrm{T}$ cells is important in the immunotherapeutic strategy of SLE treatment. Cytotoxic T lymphocytes is an important effector pathway in the pathogenesis of SLE. CD8+ T lymphocytes are activated by SLE dendritic cells into effector-type cytotoxic $\mathrm{T}$ lymphocytes, and an increased proportion of cytotoxic $\mathrm{T}$ lymphocytes correlated with SLEDAI scores [34]. A recent study found predominance of CD8+ T lymphocytes among periglomerular-infiltrating cells in the renal biopsy specimens of patients with class III/IV lupus nephritis [35]. Using immunochemistry studies, renal CD8 $+\mathrm{T}$ cell infiltration correlated with the renal activity index and high serum creatinine levels. There were also correlations with cellular crescents and Bowman's capsule rupture, and association with a poor response after conventional induction therapy.

This understanding may explain why the multitarget therapy for the treatment of class V + IV lupus nephritis was highly effective with $90 \%$ achieving complete and partial remission compared with $45 \%$ with IV CYC therapy. The effect of tacrolimus on $\mathrm{T}$ cells may be a critical component of the observed benefits in the study by Bao et al. [20] as the severe focal and segmental forms of lupus nephritis have a dominant $\mathrm{T}$ cell-dependent pathogenesis [36]. MMF, besides suppressing lymphocyte proliferation and decreasing antibodies formation, can suppress IL-2 production additionally when given with tacrolimus [37]. Hence, multitarget therapy using a combination of MMF, tacrolimus, and steroids, previously shown to be an effective treatment for early mixed cellular and humoral renal allograft rejections in the field of organ transplantation, can now be applied to the therapy of severe or resistant SLE, particularly lupus nephritis.

\section{Conclusion}

Having reviewed the experimental and clinical evidence available to date on the efficacy of tacrolimus in the treatment of SLE, it can be concluded that tacrolimus has an established role in SLE management. Immunocytokine studies support the clinical efficacy of tacrolimus through direct $\mathrm{T}$ cell suppression, inhibiting B cell activity indirectly by interfering with $\mathrm{T}$ helper signals and cytokine suppression including $\mathrm{T}_{\mathrm{H}} 1$ cytokines, IL-2, IL-6, and IL-10.

Based on current available evidence, I would recommend the usage of tacrolimus in SLE management as follows:

(1) as a steroid sparing agent;

(2) effective in SLE without renal involvement;

(3) effective in SLE with renal involvement;

(a) in class IV lupus nephritis,

(b) in class $V$ lupus nephritis,

(c) in class V + IV lupus nephritis (as multitarget therapy),

(4) as an induction therapy, maintenance therapy and also in relapsed cases as a disease remitting agent.

The use of multitarget therapy needs to be further studied and even extrapolated to the management of severe SLE patients with other organ involvements. Tacrolimus is a useful and efficacious addition to the armamentarium of SLE immunotherapy.

\section{References}

[1] C. S. Lau, "Who's afraid of the big bad wolf? Fifth Tay Chong Hai lecture of the national arthritis foundation of Singapore," APLAR Journal of Rheumatology, vol. 7, no. 1, pp. 83-89, 2004.

[2] D. A. De Albuquerque, F. M. Ebling, D. W. Lienesch, R. R. Singh, and B. H. Hahn, "Analyses of serum levels of type 1 , type 2 and type 3 cytokines reveal multiple abnormalities in lupus-prone $(\mathrm{NZB} \times \mathrm{NZW}) \mathrm{F} 1$ mice," APLAR Journal of Rheumatology, vol. 7, no. 1, pp. 3-10, 2004.

[3] G. Grondal, I. Gunnarsson, J. Ronnelid, S. Rogberg, L. Klareskog, and I. Lundberg, "Cytokine production, serum levels and disease activity in systemic lupus erythematosus," Clinical and Experimental Rheumatology, vol. 18, no. 5, pp. 565-570, 2000.

[4] K. Takabayashi, T. Koike, K. Kurasawa, et al., "Effect of FK-506, a novel immunosuppressive drug on murine systemic lupus erythematosus," Clinical Immunology and Immunopathology, vol. 51, no. 1, pp. 110-117, 1989.

[5] C. Entani, Y. Izumino, H. Iida, et al., "Effect of a novel immunosuppressant, FK506, on spontaneous lupus nephritis in MRL/MpJ-lpr/lpr mice," Nephron, vol. 64, no. 3, pp. 471475, 1993.

[6] M. Duddridge and R. J. Powell, "Treatment of severe and difficult cases of systemic lupus erythematosus with tacrolimus: a report of three cases," Annals of the Rheumatic Diseases, vol. 56, no. 11, pp. 690-692, 1997.

[7] S. L. Walker, B. Kirby, and R. J. G. Chalmers, "The effect of topical tacrolimus on severe recalcitrant chronic discoid lupus erythematosus," British Journal of Dermatology, vol. 147, no. 2, pp. 405-406, 2002.

[8] T. Kanekura, N. Yoshii, K. Terasaki, H. Miyoshi, and T. Kanzaki, "Efficacy of topical tacrolimus for treating the malar rash of systemic lupus erythematosus," British Journal of Dermatology, vol. 148, no. 2, pp. 353-356, 2003. 
[9] V. Madan, P. J. August, and R. J. Chalmers, "Efficacy of topical tacolimus $0.3 \%$ in clobetasol propionate $0.05 \%$ ointment in therapy-resistant cutaneous lupus erythematosus: a cohort study," Clinical and Experimental Dermatology, vol. 35, no. 1, pp. 27-30, 2010.

[10] K. Yoshie, T. Nahoko, K. Kaichi, et al., "Tacrolimus therapy for systemic lupus erythematosus without renal involvement: a preliminary retrospective study," Modern Rheumatology, vol. 19, no. 6, pp. 329-333, 2009.

[11] K. H. Yoon, "Successful usage of tacrolimus (FK506) in resistant/relapsed rheumatic diseases," APLAR Journal of Rheumatology, vol. 7, no. 1, pp. 44-48, 2004.

[12] C. L. Suzanne, K. Yukiko, L. Kenneth, et al., "Treatment of pediatric patients with severe systemic lupus erythematosus with tacrolimus," Annals of Rheumatic Diseases, supplement, pp. 48-49, 2003.

[13] C. C. Mok, K. H. Tong, C. H. To, Y. P. Siu, and T. C. $\mathrm{Au}$, "Tacrolimus for induction therapy of diffuse proliferative lupus nephritis: an open-labeled pilot study," Kidney International, vol. 68, no. 2, pp. 813-817, 2005.

[14] H. T. Zhang, W. X. Hu, H. L. Xie, et al., “Tacrolimus versus intravenous cyclophosphamide in the induction therapy of diffuse proliferative lupus nephritis," Nephrology Dialysis Transplantation, vol. 15, pp. 501-507, 2006.

[15] M. Maruyama, Y. Yamasaki, K. Sada, et al., "Good response of membranous lupus nephritis to tacrolimus," Clinical Nephrology, vol. 65, no. 4, pp. 276-279, 2006.

[16] C.-C. Szeto, B. C.-H. Kwan, F. M.-M. Lai, et al., "Tacrolimus for the treatment of systemic lupus erythematosus with pure class V nephritis," Rheumatology, vol. 47, no. 11, pp. 1678$1681,2008$.

[17] K. C. Tse, M. F. Lam, S. C. W. Tang, C. S. O. Tang, and T. M. Chan, "A pilot study on tacrolimus treatment in membranous or quiescent lupus nephritis with proteinuria resistant to angiotensin inhibition or blockade," Lupus, vol. 16, no. 1, pp. 46-51, 2007.

[18] A. Yukari, U. Keiko, O. Shigeru, et al., "Clinical assessment of tacrolimus therapy in lupus nephritis: one-year follow-up study in a single center," Nephron Clinical Practice, vol. 113, pp. 330-336, 2009.

[19] M. Nobuyuki, K. Shinichi, and H. Hiroshi, "Efficacy and safety of tacrolimus for lupus nephritis: a placebo-controlled doubleblind multicenter study," Modern Rheumatology, vol. 19, no. 6, pp. 606-615, 2009.

[20] H. Bao, Z. H. Liu, H.-L. Xie, W.-X. Hu, H.-T. Zhang, and L.-S. Li, "Successful treatment of class V+IV lupus nephritis with multitarget therapy," Journal of the American Society of Nephrology, vol. 19, no. 10, pp. 2001-2010, 2008.

[21] H. T. Zhang, W. X. Hu, H. L. Xie, et al., "Randomized controlled trial of tacrolimus versus intravenous cyclophosphamide in the induction therapy of class V plus IV lupus nephritis," Nephrology Dialysis Transplantation, vol. 15, pp. 508-514, 2006.

[22] K. S. Peterson and R. Winchester, "Systemic lupus erythematosus," in Pathogenesis, J. K. William and W. M. Larry, Eds., pp. 1523-1559, Lippincott Williams \& Wilikins, Philadelphia, Pa, USA, 2005.

[23] K. A. Kirou and M. K. Crow, "New pieces to the SLE cytokine puzzle," Clinical Immunology, vol. 91, no. 1, pp. 1-5, 1999.

[24] L. C. Ginus, A. B. Cosim, and P. J. Morris, Transplantation, Blackwell Science, Oxford, UK, 1999.

[25] S. Sakuma, Y. Kato, F. Nishigaki, et al., "FK506 potently inhibits T cell activation induced TNF- $\alpha$ and IL- $1 \beta$ production in vitro by human peripheral blood mononuclear cells,"
British Journal of Pharmacology, vol. 130, no. 7, pp. 1655-1663, 2000.

[26] S. Sakuma, Y. Higashi, N. Sato, et al., "Tacrolimus suppressed the production of cytokines involved in atopic dermatitis by direct stimulation of human PBMC system. (Comparison with steroids)," International Immunopharmacology, vol. 1, no. 6, pp. 1219-1226, 2001.

[27] K. Magari, S. Miyata, F. Nishigaki, Y. Ohkubo, S. Mutoh, and T. Goto, "Differential effects of FK506 and methotrexate on inflammatory cytokine levels in rat adjuvant-induced arthritis," Journal of Rheumatology, vol. 30, no. 10, pp. 21932200, 2003.

[28] S. Sakuma, Y. Kato, F. Nishigaki, et al., "Effects of FK506 and other immunosuppressive anti-rheumatic agents on $\mathrm{T}$ cell activation mediated IL-6 and IgM production in vitro," International Immunopharmacology, vol. 1, no. 4, pp. 749-757, 2001.

[29] Y. Furukawa, H. Yoshikawa, K. Iwasa, and M. Yamada, "Clinical efficacy and cytokine network-modulating effects of tacrolimus in myasthenia gravis," Journal of Neuroimmunology, vol. 195, no. 1-2, pp. 108-115, 2008.

[30] M. Sugiyama, M. Funauchi, T. Yamagata, et al., "Predominant inhibition of Th1 cytokines in New Zealand black/white F1 mice treated with FK506," Scandinavian Journal of Rheumatology, vol. 33, no. 2, pp. 108-114, 2004.

[31] H. Jiang, C. Wynn, F. Pan, A. Ebbs, L. M. Erickson, and M. Kobayashi, "Tacrolimus and cyclosporine differ in their capacity to overcome ongoing allograft rejection as a result of their differential abilities to inhibit interleukin-10 production," Transplantation, vol. 73, no. 11, pp. 1808-1817, 2002.

[32] L. Llorente and Y. Richaud-Patin, "The role of interleukin-10 in systemic lupus erythematosus," Journal of Autoimmunity, vol. 20, no. 4, pp. 287-289, 2003.

[33] S. Heidt, D. L. Roelen, C. Eijsink, et al., "Calcineurin inhibitors affect B cell antibody responses indirectly by interfering with T cell help," The Journal of Translational Immunology, vol. 159, no. 2, pp. 199-207, 2009.

[34] P. Blanco, V. Pitard, J.-F. Viallard, J.-L. Taupin, J.-L. Pellegrin, and J.-F. Moreau, "Increase in activated $\mathrm{CD}^{+} \mathrm{T}$ lymphocytes expressing perforin and granzyme B correlates with disease activity in patients with systemic lupus erythematosus," Arthritis and Rheumatism, vol. 52, no. 1, pp. 201-211, 2005.

[35] L. Couzi, P. Merville, C. Deminiere, et al., "Predominance of $\mathrm{CD}^{+} \mathrm{T}$ lymphocytes among periglomerular infiltrating cells and link to the prognosis of class III and class IV lupus nephritis," Arthritis and Rheumatism, vol. 56, no. 7, pp. 23622370, 2007.

[36] R. J. Glassock, "Multitarget therapy of lupus nephritis: base hit or home run?" Journal of the American Society of Nephrology, vol. 19, no. 10, pp. 1842-1844, 2008.

[37] O. Millan, M. Brunet, J. M. Campistol, et al., "Pharmacodynamic approach to immunosuppressive therapies using calcineurin inhibitors and mycophenolate mofetil," Clinical Chemistry, vol. 49, no. 11, pp. 1891-1899, 2003. 


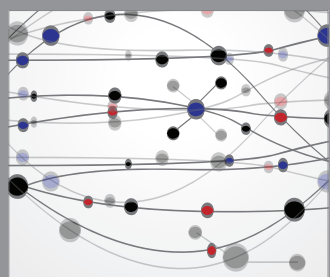

The Scientific World Journal
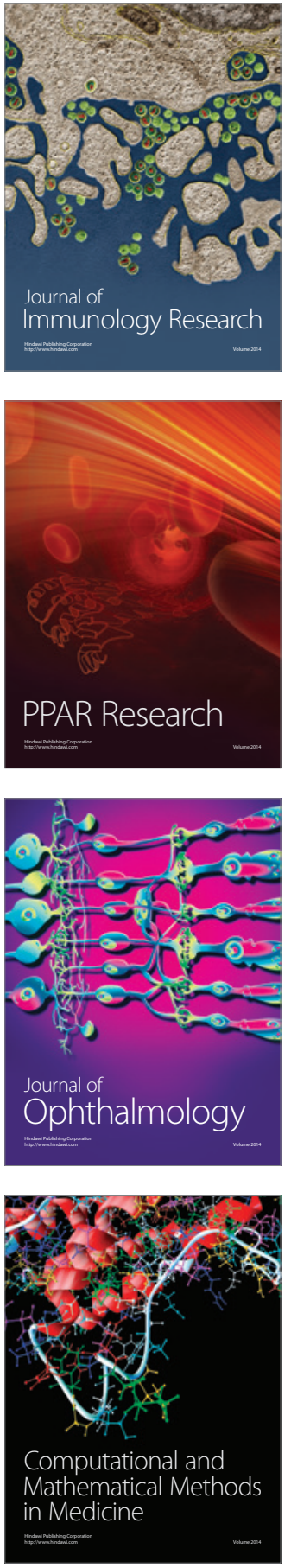

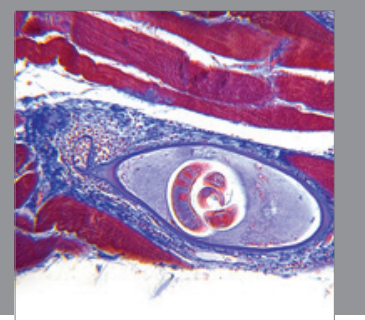

Gastroenterology

Research and Practice
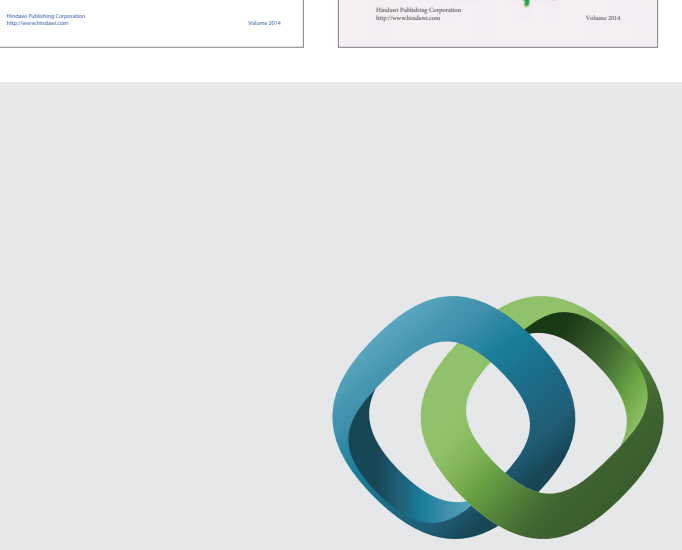

\section{Hindawi}

Submit your manuscripts at

http://www.hindawi.com
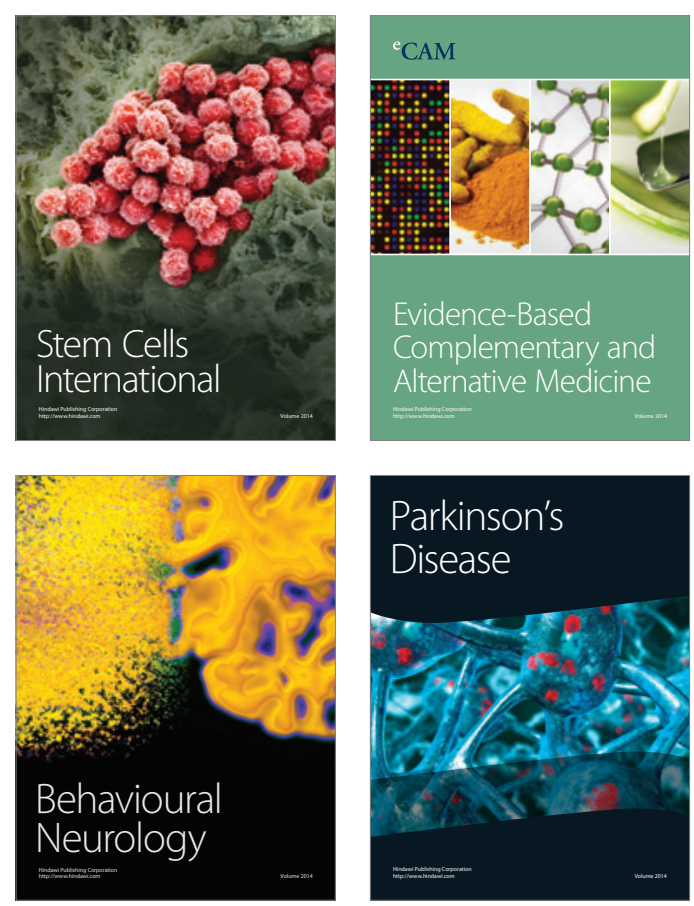

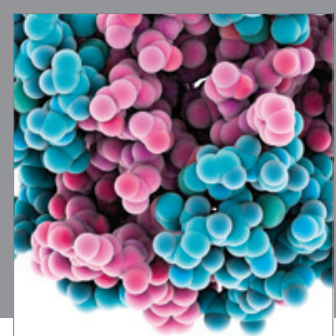

Journal of
Diabetes Research

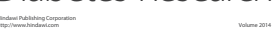

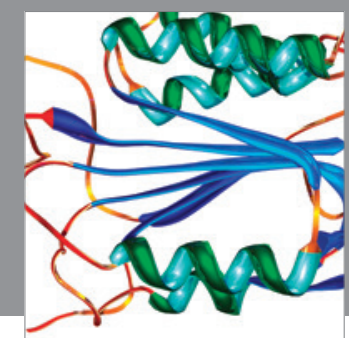

Disease Markers
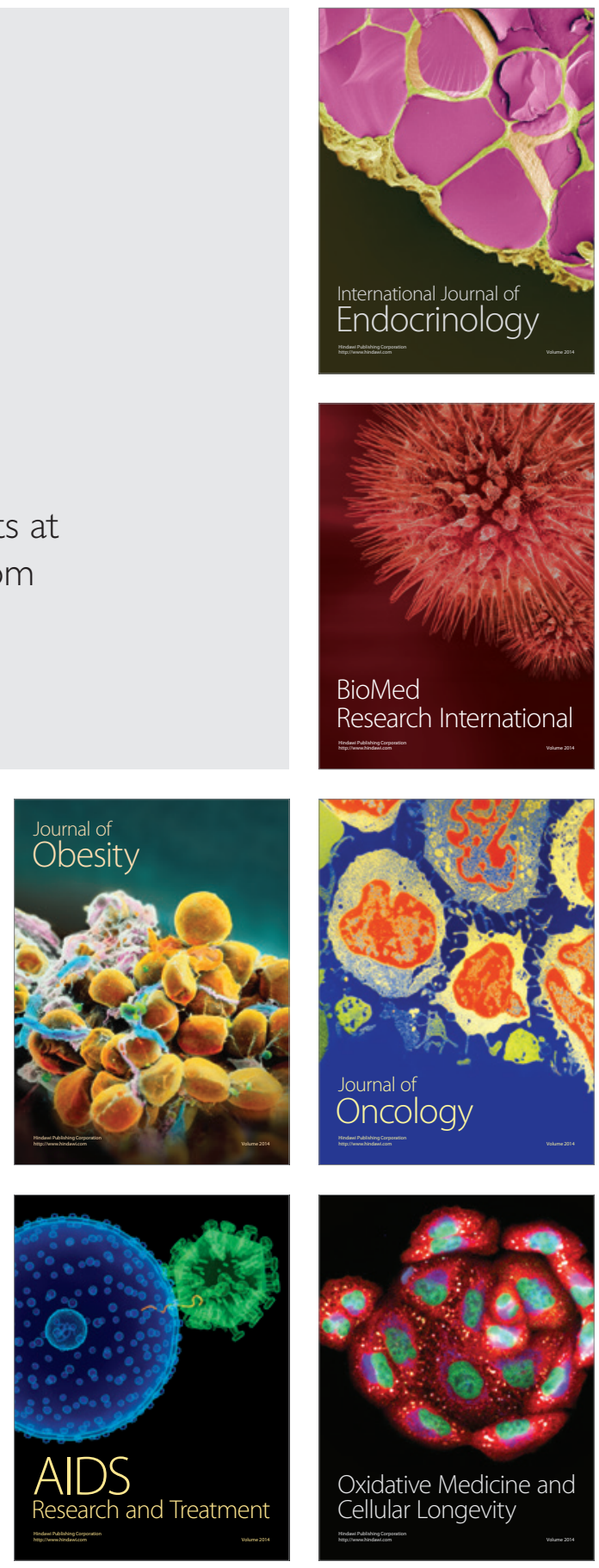